This item was submitted to Loughborough's Institutional Repository (https://dspace.lboro.ac.uk/) by the author and is made available under the following Creative Commons Licence conditions.

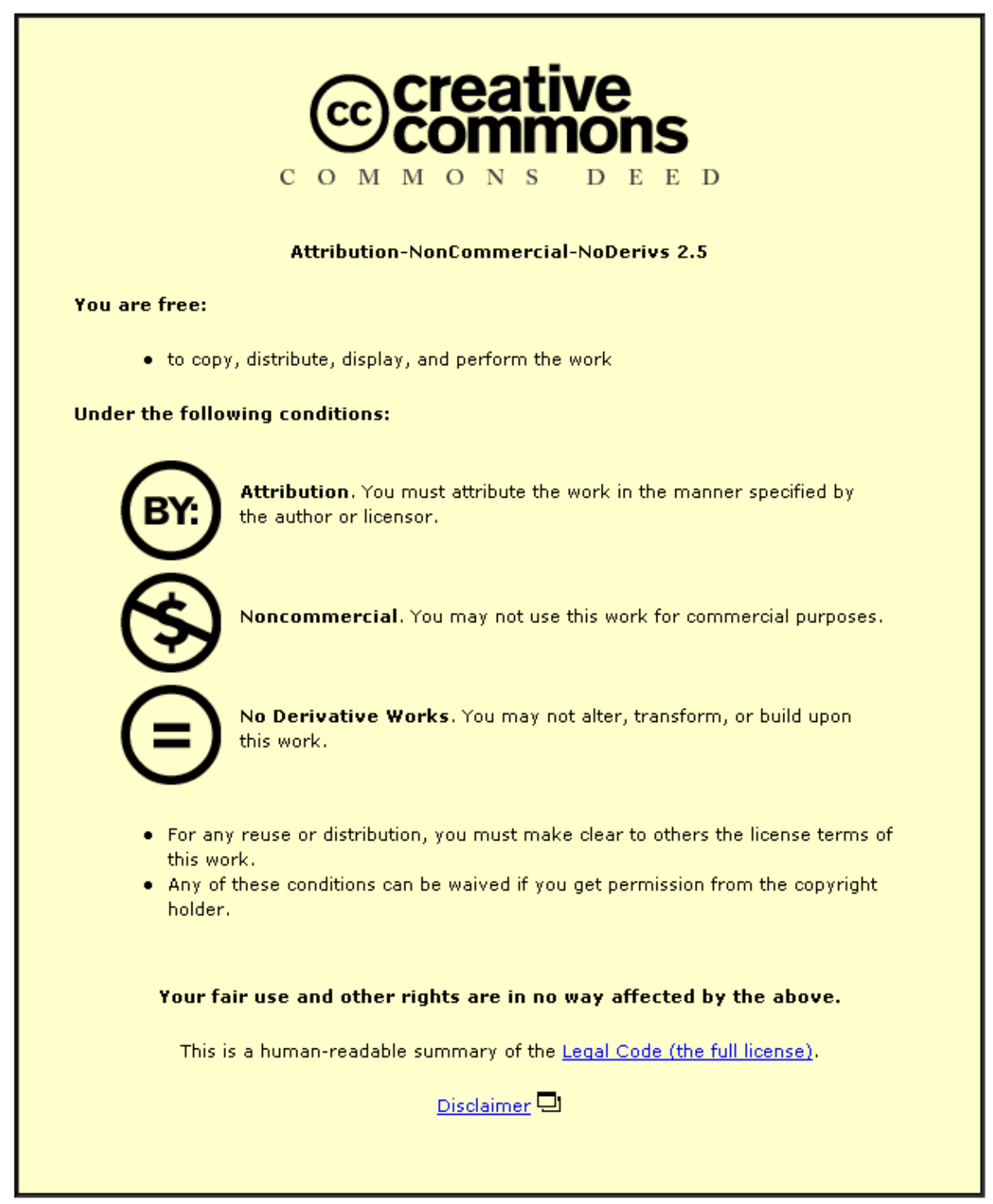

For the full text of this licence, please go to: http://creativecommons.org/licenses/by-nc-nd/2.5/ 
Journal of Applied Biomechanics, 20, 309-316, 2004

\title{
Wobbling mass influence on impact ground reaction forces: A simulation model sensitivity analysis
}

\author{
Matthew T. G. Pain ${ }^{1}$ and John H. Challis ${ }^{2}$ \\ ${ }^{1}$ School of Sport and Exercise Sciences, Loughborough University, United Kingdom \\ ${ }^{2}$ Biomechanics Laboratory, The Pennsylvania State University, PA 16802-3408, USA
}

\begin{abstract}
To gain insight into joint loadings during impacts, wobbling mass models have been used. The aim of this study was to investigate the sensitivity of a wobbling mass model, of landing from a drop, to the model's parameters. A two-dimensional wobbling mass model was developed. Three rigid linked segments designed to represent the skeleton each had a second mass attached to them, via two translational non-linear spring dampers, representing the soft tissue. Model parameters were systematically varied one at a time and the effect this had on the peak vertical ground reaction force and segment kinematics was examined. Model output showed low sensitivity to most model parameters but was sensitive to the timing of joint torque initiation. Varying the heel pad stiffness in the range of stiffness values reported in the literature had the largest influence on the peak vertical ground reaction force. The analysis indicated that the more proximal body segments had a lower influence on peak vertical ground reaction force per unit mass than the segments nearer the contact point, $340 \mathrm{~N} / \mathrm{kg}, 157 \mathrm{~N} / \mathrm{kg}$ and $24 \mathrm{~N} / \mathrm{kg}$ for the shank, thigh and trunk respectively. Model simulations were relatively insensitive to variations in the properties of the connection between the wobbling masses and the skeleton. Given the proviso that estimates for the other model parameters and joint torque activation timings lie in a realistic range, then if the goal is to examine the effects of the wobbling mass on the system this insensitivity is an advantage. If precise knowledge about the motion of the wobbling mass is of interest, however, more experimental work is required to determine precisely these model parameters.
\end{abstract}

Keywords: mass distribution, heel pad, activation timing

\section{INTRODUCTION}

Biomechanical whole body models are normally composed of rigid segments linked by simple kinematic connections (e.g. Bobbert \& van Soest, 1994; Gerritsen, van den Bogert, \& Nigg, 1995). However segments of the human body are not rigid and such an assumption can lead to substantial errors in both inverse and direct dynamics analyses as they ignore intrasegmental forces and their effect (Pain \& Challis 2002). Quantifying, via modeling, the influence of soft tissue motion on the kinematics and kinetics of human motion may provide important insights into the loading of the human body.

Cole, Nigg, van den Bogert, \& Gerritsen, (1996) and Gruber, Ruder, Denoth, \& Schneider, (1998) developed wobbling mass models to examine joint loading during impacts. Cole et al. (1996) had a restricted set of wobbling mass parameters. The wobbling masses were point masses constricted to move along the line of action of the muscle-tendon unit and had a moment of inertia of zero. Gruber et al. (1998) compared a wobbling mass model with a rigid segment model for simulating landing from a drop. Peak resultant joint moments were lower for the wobbling mass model compared with a rigid model. However neither study investigated the sensitivity of the results to the model parameter values

In Gruber et al. (1998) the validation of the model consisted of matching the ground reaction forces between model and subject. However a large number of model parameters were optimized to achieve the ground reaction force match. The sensitivity of the results to variations in the model parameters was not examined. The distributions of the segmental mass between skeletal and soft tissue component were well beyond the ranges indicated from dissection by Clarys, Martin, \& Drinkwater, (1984). Model joint torques were zero until five 
milliseconds after impact, but inverse dynamics analysis of landings show significant joint torques prior to impact (Bobbert, Yeadon, \& Nigg, 1992). Such anomalies suggest that compensating errors may have occurred in their simulations.

Nigg \& Liu (1999) and Liu \& Nigg (2000) presented a model to investigate the impact phase of running. Their model considered vertical motion only, it had two bodies with rigid and wobbling masses components connected with linear springs and dampers. They performed a limited sensitivity analysis and illustrated the sensitivity of predicted vertical ground reaction force to variations in model parameters. Liu \& Nigg (2000, page 219) concluded that future research should "take into consideration the influence of individual variation in lower body masses and mass distributions". If multi-segment wobbling mass models are to be used to investigate the kinematics and kinetics of human impacts then the potential contribution of each model parameter, within physiological ranges, needs to be examined. The aim of this study was to investigate the sensitivity of a two-dimensional multi-segment wobbling mass model of landing from a drop to the model parameters.

\section{METHODS}

A two-dimensional wobbling mass model was developed in DADS (version 9.00, CADSI) to simulate landing from a drop of $40 \mathrm{~cm}$ onto the heels. The task selected here parallels that used by Gruber et al. (1998), and reflects a condition which can occur during landings from a jump, especially somersaults, and provides links to a running where most impacts are via the heel. The model consisted of three body segments: one representing the head, arms and trunk segments: the second representing the upper leg: and the third representing the shank and foot. The rigid links were connected with revolute joints equipped with controllable joint actuators to emulate muscle actions. Each body segment consisted of a rigid component representing the skeleton which had a second mass attached to it, representing the wobbling mass (Figure 1). The connection between the two was via two non-linear spring dampers, which represent the gross force interaction. For further details of a model body segment see Pain \& Challis (2001). This connection will be referred to as a "tendon", but does not strictly represent the mechanical properties of the tendons only. The equation representing the properties of these tendons is:

$$
\mathrm{F}_{\mathrm{T}}=\mathrm{K}_{\mathrm{T}} \cdot \Delta \mathrm{L}_{\mathrm{T}}^{3}-\mathrm{C}_{\mathrm{T}} \cdot \dot{\mathrm{L}}_{\mathrm{T}}
$$

where $\mathrm{F}_{\mathrm{T}}$ is the restoring force in the "tendon" connecting the wobbling mass to the skeleton, $\mathrm{K}_{\mathrm{T}}$ is the stiffness coefficient relating length changes to "tendon" force, $\Delta \mathrm{L}_{\mathrm{T}}$ is the change in length of the "tendon", $\mathrm{C}_{\mathrm{T}}$ is the damping coefficient of the "tendon", and $\dot{\mathrm{L}}_{\mathrm{T}}$ is the rate of change of the length of "tendon" with respect to time.

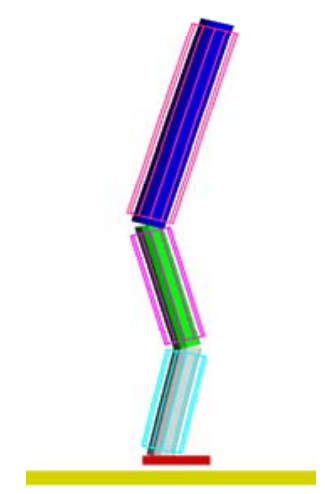

Figure 1. The three-link wobbling mass model just before impact. Inner solid segments represent the rigid skeleton. The outer line segments represent the wobbling mass material. 
The ground-heel interface was represented by a non-linear spring-damper system that had similar mechanical properties to those of simulated human heel pads used in other models (e.g. Güler, Berme, \& Simon, 1998). The equation representing the properties of the heel pad is:

$$
\mathrm{F}_{\mathrm{H}}=\mathrm{K}_{\mathrm{H}} \cdot \Delta \mathrm{L}_{\mathrm{H}}^{2.5}-\mathrm{C}_{\mathrm{H}} \cdot \dot{\mathrm{L}}_{\mathrm{H}}
$$

where $\mathrm{F}_{\mathrm{H}}$ is the heel pad force, $\mathrm{K}_{\mathrm{H}}$ is the stiffness coefficient relating length changes to heel pad force, $\Delta \mathrm{L}_{\mathrm{H}}$ is the deformation of the heel pad, $\mathrm{C}_{\mathrm{H}}$ is damping coefficient of the heel pad, and $\dot{\mathrm{L}}_{\mathrm{H}}$ is the rate of change of heel pad deformation with respect to time. The heel pad and tendon model parameters are presented in Table 1.

Table 1. Model inertial parameters and coefficients for the equations describing the non-linear spring dampers connecting the wobbling and skeletal masses, and the heel pad element.

\begin{tabular}{|c|c|c|c|c|c|c|}
\hline $\begin{array}{c}\text { Model } \\
\text { Segment }\end{array}$ & $\begin{array}{c}\text { Stiffness } \\
\text { coefficient } \\
\left(\mathrm{K}_{\mathrm{T}}\right) \\
\left(\mathrm{kg} \cdot \mathrm{m}^{-2} \cdot \mathrm{s}^{-2}\right. \\
\left.\mathrm{x} 10^{8}\right)\end{array}$ & $\begin{array}{c}\text { Damping } \\
\text { coefficient } \\
\left(\mathrm{C}_{\mathrm{T}}\right) \\
\left(\mathrm{N} . \mathrm{s}^{-1}\right)\end{array}$ & $\begin{array}{c}\text { Bone } \\
\text { Mass } \\
(\mathrm{kg})\end{array}$ & $\begin{array}{l}\text { Soft tissue } \\
\text { mass } \\
(\mathrm{kg})\end{array}$ & $\begin{array}{c}\text { Bone } \\
\text { moment of } \\
\text { inertia } \\
\left(\mathrm{kg} \mathrm{m}^{2}\right)\end{array}$ & $\begin{array}{c}\text { Soft tissue } \\
\text { moment of } \\
\text { inertia } \\
\left(\mathrm{kg} \mathrm{m}^{2}\right)\end{array}$ \\
\hline Shank & 0.9 & 280 & 3.0 & 2.6 & 0.029 & 0.063 \\
\hline Thigh & 1.8 & 560 & 5.3 & 10.5 & 0.059 & 0.126 \\
\hline Torso & 2.3 & 700 & 20.9 & 20.9 & 0.505 & 0.950 \\
\hline Heel pad & 40 & 2500 & & & & \\
\hline
\end{tabular}

Anthropometric parameters, initial body configuration, and joint torque profiles were the same as those presented in Gruber et al. (1998) as they had produced a successful simulation of landing. Gruber et al. (1998) only initiated their torque actuators $5 \mathrm{~ms}$ after impact, here they were also initiated either at impact, or 5ms before. Only the first $100 \mathrm{~ms}$ of the landing were simulated, paying particular attention to the passive impact phase.

Sensitivity analysis was performed by varying one model parameter at a time and quantifying the effect this had on peak vertical ground reaction force (PVGRF) and segment kinematics using a kinematic exemplar of thigh angle $40 \mathrm{~ms}$ post impact. These two variables were chosen as they are indicative of the severity of an impacting landing. Sensitivity analyses were performed for each of the actuator timings.

The majority of perturbations were $\pm 20 \%$ of the initial values. Error in measured or calculated inertial parameters and mass distributions should not be greater than this for personalized measurements (e.g. Clarys and Marfell-Jones, 1986; Yeadon, 1990). Heel pad mechanical properties measured in vivo and in vitro are accurate and repeatable (Aerts, Ker, De Clercq, Ilsley, \& Alexander, 1995), but estimates of in vivo and in vitro stiffness vary by an order of magnitude (e.g. Valiant, 1984; Bennett \& Ker, 1990). For the sensitivity analyses heel pad properties reflecting mechanical properties measured in vivo and in vitro were used. No experimental data exist to justify the properties of the model's tendons however they are a composite of tendon, muscle and other soft tissue and the stiffness value of muscle can vary considerably. In both these cases variations of one order of magnitude were also carried out while near critical damping was maintained, (Pain \& Challis 2002). 


\section{RESULTS}

The kinematics of the model for the first $40 \mathrm{~ms}$ of impact were relatively insensitive to variations in the model parameters (Table 2). The kinetics of the model for the first $40 \mathrm{~ms}$ of impact were more sensitive to variations in model parameters. Changing the heel pad stiffness by an order of magnitude had the greatest effect on the ground reaction forces but not on the kinematics. Changes in the ratio of the soft tissue mass to bone mass was the most sensitive wobbling mass parameter giving a change in PVGRF of $+13 \%$ for an increase in bone mass of $20 \%$, and a similar decrease when this ratio was decreased (Table 2). Tendon stiffness was the least sensitive parameter examined.

Table 2. Percent change in peak vertical ground reaction force (VGRF) and thigh angle for variation in model parameters with joint actuators initiated $5 \mathrm{~ms}$ before and $5 \mathrm{~ms}$ after impact.

\begin{tabular}{|c|c|c|c|c|}
\hline \multirow[b]{2}{*}{$\begin{array}{c}\text { Model Parameter } \\
\text { varied }\end{array}$} & \multicolumn{2}{|c|}{$\begin{array}{l}\text { Joint Torques Initiated } \\
5 \text { ms after Impact. }\end{array}$} & \multicolumn{2}{|c|}{$\begin{array}{l}\text { Joint Torques Initiated } \\
5 \text { ms before Impact. }\end{array}$} \\
\hline & $\begin{array}{c}\% \Delta \\
\text { Peak } \\
\text { VGRF }\end{array}$ & $\begin{array}{c}\% \Delta \\
\text { Thigh } \\
\text { Angle }\end{array}$ & $\begin{array}{c}\% \Delta \\
\text { Peak } \\
\text { VGRF }\end{array}$ & $\begin{array}{c}\% \Delta \\
\text { Thigh } \\
\text { Angle }\end{array}$ \\
\hline Bone mass $+20 \%$ & +13.0 & +1.0 & +11.0 & +1.4 \\
\hline Bone mass - $20 \%$ & -12.9 & -0.7 & -12.5 & -0.0 \\
\hline Tendon $\mathrm{K}+20 \%$ & -0.1 & 0.0 & -0.0 & +0.6 \\
\hline Tendon $\mathrm{K} \times 10$ & +1.2 & +0.5 & +0.2 & +2.0 \\
\hline Heel K + 20\% & +5.0 & +0.2 & +5.0 & +0.4 \\
\hline Heel K - 20\% & -5.0 & -0.4 & -5.5 & -0.2 \\
\hline Heel damping $+20 \%$ & +4.0 & 0.0 & +3.3 & 0.0 \\
\hline Heel damping - 20\% & -4.0 & 0.0 & -3.6 & 0.0 \\
\hline $\begin{array}{c}\text { Heel K, damping }+ \\
20 \%\end{array}$ & +0.1 & +0.2 & +0.8 & +0.4 \\
\hline Heel K, damping - 20\% & -3.0 & -0.3 & -1.8 & -0.2 \\
\hline Heel $\mathrm{K}$, damping $\times 10$ & +91.2 & +1.6 & +80.3 & +2.2 \\
\hline Heel K, damping / 10 & -25.1 & -5.5 & -28.3 & -6.7 \\
\hline
\end{tabular}

The model was sensitive to the timing of production of the joint torques (Figure 2). The vertical ground reaction forces estimated by the model using the original parameter set with torques initiated $5 \mathrm{~ms}$ after impact are presented in Figure 2a. The figure shows a pattern typical for landing from a drop, which is similar to that reported by Gruber et al. (1998). The PVGRF and the thigh angle $40 \mathrm{~ms}$ after impact differed for torques initiated at impact and 5 ms before impact compared with torques initiated $5 \mathrm{~ms}$ after impact (Table 3). The time of peak force was the same in all conditions. PVGRF increased by $28 \%$ when the joint torques were initiated 5 ms before impact, compared with initiation $5 \mathrm{~ms}$ after impact. 


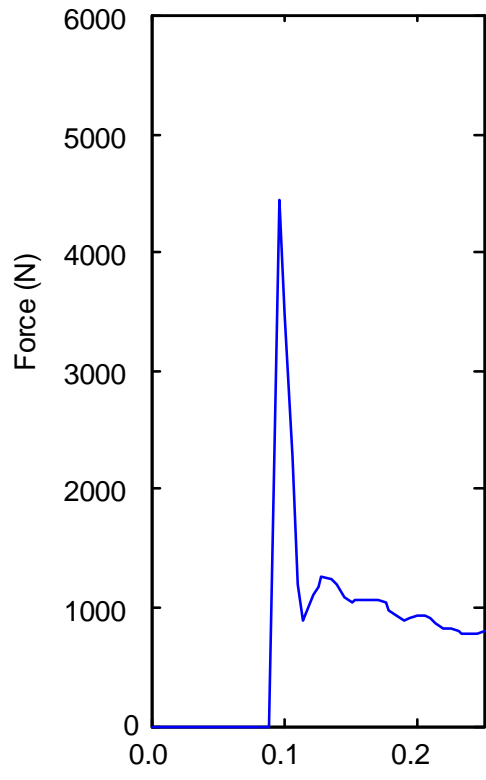

(a)

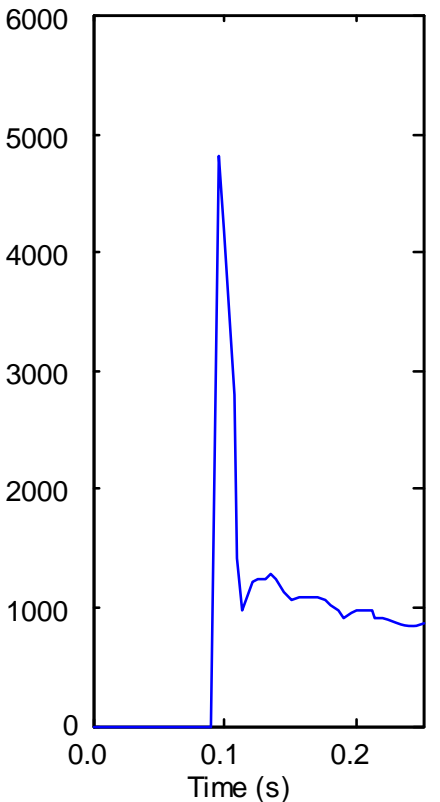

(b)

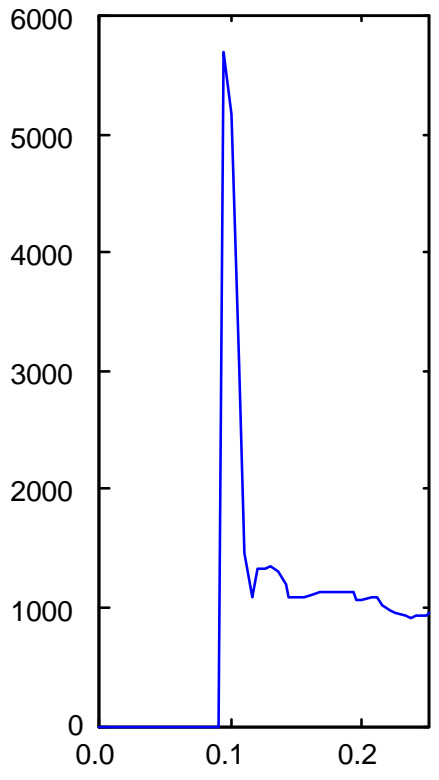

(c)

Figure 2. Vertical ground reaction force time history obtained with three torque activation timings: (a) torque initiated 5 ms after impact; (b) torque initiated at impact; (c) torque initiated $5 \mathrm{~ms}$ before impact.

Table 3. Percent change in peak vertical ground reaction force (VGRF) and thigh angle $40 \mathrm{~ms}$ after landing. The values obtained when torques are initiated 5 ms before impact and at impact, are compared with values obtained when torques are initiated $5 \mathrm{~ms}$ after impact.

\begin{tabular}{|c|c|c|}
\hline $\begin{array}{c}\text { Joint Torques } \\
\text { Initiated }\end{array}$ & $\% \Delta$ Peak VGRF & \\
\hline 5 ms before impact. & +28.0 & +7.2 \\
\hline At impact & +8.5 & +3.1 \\
\hline
\end{tabular}

Initiating the joint actuators $5 \mathrm{~ms}$ before impact tended to slightly reduce the effects of other parameter variations on the PVGRF but increased them on the kinematic variations. Increasing the mass of the bone of the shank, thigh and torso individually by $20 \%$ gave increases of $3 \%, 5 \%$ and $2 \%$ respectively in the PVGRF.

\section{DISCUSSION}

The study investigated the sensitivity of landing kinematics and kinetics for a multisegment wobbling mass model of landing from a drop. Changing the ratio of soft tissue to bone mass had one of the more considerable effects on the impact force, and segment kinematics. This is unsurprising as the initial impact peak is mainly due to deceleration of the rigid body skeleton from force applied to the calcaneous from the ground-heel pad interaction. Expressing the change in PVGRF per kilogram of mass change gives: $340 \mathrm{~N} / \mathrm{kg}, 157 \mathrm{~N} / \mathrm{kg}$ and $24 \mathrm{~N} / \mathrm{kg}$ for the shank, thigh and trunk respectively. The shank segment had the greatest influence on the PVGRF per unit mass.

Measurements of heel pad properties vary by orders of magnitude (e.g. Valiant, 1984; Bennett \& Ker, 1990). The results demonstrated that variations of heel pad stiffness of this 
magnitude affect the PVGRF more than any other perturbation. The selection of a valid heel pad component is necessary to avoid compensating errors in other model components when attempting to recreate vertical ground reaction forces. Pain and Challis (2001) have shown that some heel pad models effectively accommodate the influence of shank soft tissue motion, this approach may be appropriate but should be acknowledged.

The timing of the joint torques is important because stiffer joints increase the effective mass of the rigid body by not allowing body segments to freely rotate. Another possible effect is the active acceleration of a segment into the impact or away from it. This may be more significant in actively soft landings. Having no stiffness in the joints until after impact has begun, as used in the simulation model of Gruber et al. (1998), is highly unlikely as studies have shown that to control a landing effectively the knee musculature has to be highly activated pre-landing (Bobbert et al., 1992).

Stiffness in the joints slightly reduces the effects of varying the other parameters on the vertical ground reaction force but increases kinematic variations. By having a stiffer system impacting, the mass of each rigid body becomes more dominant due to its larger effective mass. In this case the segments further from the contact point will have a greater influence on the initial impact, but these have been shown to have less effect per unit mass on the vertical ground reaction force. So although the PVGRF will be greater than for less stiff joints, the sensitivity to variations will be less. With the stiffer joints the impacting forces can be distributed to more adjacent rigid segments than to the wobbling mass of that segment. Thus varying the wobbling mass parameters will not have such a great effect on the rigid segment kinematics.

The bone mass and the joint torque timing were the most sensitive parameters found in this study. The model of Gruber et al. (1998) should have similar sensitivities. The mass distribution data from Clarys \& Marfell-Jones (1986) would suggest that the rigid segment mass (the bone) is much less than that used in Gruber et al. (1998). It seems likely that the model of Gruber et al. (1998) has some compensating errors in these parameters: that is overly heavy bone segments accommodate for the lack of structural stiffness from initiating the joint torques after impact. Accurate measures of subject specific inertial parameters and mass distributions are necessary for wobbling mass models and would ideally be obtained from MRI scans.

Model simulations were relatively insensitive to variations in tendon parameters. If the goal is to examine the effects of the wobbling mass on the system then this insensitivity is an advantage. If precise knowledge about the motion of the wobbling mass is of interest, however, more experimental work is required to determine precisely these model parameters. To produce an accurate wobbling mass model of an impact the correct heel pad properties, in vitro measures, accurate mass distributions and effective joint stiffnesses, activation timing, need to be calculated as the model is most sensitive to these parameters.

\section{REFERENCES}

Aerts, P., Ker, R.F., De Clercq, D., Ilsley, D.W., \& Alexander,R. McNeill. (1995). The mechanical properties of the human heel pad: a paradox resolved. Journal of Biomechanics, 28, 1299-1308.

Bennett, M.B., \& Ker, R.F. (1990). The mechanical properties of the human subcalcaneal fat pad in compression. Journal of Anatomy, 171, 131-138.

Bobbert, M.F., Yeadon, M.R., \& Nigg, B.N. (1992). Mechanical analysis of the landing phase in heel-toe running. Journal of Biomechanics, 25, 223-234.

Bobbert, M.F., \& van Soest, A.J. (1994). Effects of muscle strengthening on vertical jump height: a simulation study. Medicine and Science in Sports and Medicine, 26, 10121020 . 
Clarys, J.P., \& Marfell-Jones, M.J. (1986). Anthropometric prediction of component tissue masses in the minor limbs of the human body. Human Biology, 58, 761-769.

Clarys, J.P., Martin, A.D., \& Drinkwater, D.T. (1984). Gross tissue weights in the human body by cadaver dissection. Human Biology, 54, 459-473.

Cole, G.K., Nigg, B.M., van den Bogert, A.J., \& Gerritsen, K.G.M. (1996). Lower extremity joint loading during impact in running. Clinical Biomechanics, 11, 181-193.

Gerritsen, K.G.M., van den Bogert, A.J., \& Nigg, B.M. (1995). Direct dynamics simulation of the impact phase in heel-toe running. Journal of Biomechanics, 28, 661-668.

Gruber, K., Ruder, H., Denoth, J., \& Schneider, K. (1998). A comparative study of impact dynamics: wobbling mass model versus rigid body model. Journal of Biomechanics, 31, 439-444.

Güler, H.S., Berme, N., \& Simon, S.R. (1998). A visco-elastic sphere model for the representation of plantar soft tissue during simulations. Journal of Biomechanics, 31, 847-853.

Ker, R.F., Bennet, M.B., Kester, R.C., \& Alexander, R. McNeill. (1989). Foot strike and the properties of the human heel pad. Proceedings of the Institute of Mechanical Engineers, 203, 191-196.

Liu, W. \& Nigg, B.M., (2000). A mechanical model to determine the influence of masses and mass distribution on the impact force during running. Journal of Biomechanics, 33, 219-224.

Nigg, B.M., \& Liu, W. (1999). The effect of muscle stiffness and damping on simulated impact forces during running. Journal of Biomechanics, 32, 849-856.

Pain, M.T.G., \& Challis, J.H. (2001). The role of the heel pad and shank soft tissue during impacts: A further resolution of a paradox. Journal of Biomechanics, 34, 327-333 .

Pain, M.T.G., \& Challis, J.H. (2002). Soft tissue motion during impacts: their potential contribution to energy dissipation. Journal of Applied Biomechanics, 18, 231-242

Valiant, G.A. (1984). A determination of the mechanical characteristics of the human heel pad in vivo. Unpublished doctoral thesis, The Pennsylvania State University, University Park.

Yeadon, M. R. (1990). The simulation of aerial movement - II. A mathematical inertia model of the human body. Journal of Biomechanics. 23, 67-74. 